\title{
Transformation and fate of natural estrogens and their conjugates in wastewater treatment plants: Influence of operational parameters and removal pathways
}

\author{
Weiwei Ben ${ }^{a}$, Bing Zhu ${ }^{\text {b }}$, Xiangjuan Yuan ${ }^{\text {a }}$, Yu Zhang a , Min Yang a , Zhimin Qiang a, * \\ ${ }^{a}$ Key Laboratory of Drinking Water Science and Technology, Research Center for Eco-Environmental Sciences, University of Chinese Academy of Sciences, \\ Chinese Academy of Sciences, 18 Shuang-qing Road, Beijing 100085, China \\ ${ }^{\mathrm{b}}$ Beijing Enterprises Water Group of China Co., Ltd., 101 Baiziwan, Beijing 100124, China
}

\section{A R T I C L E I N F O}

\section{Article history:}

Received 27 April 2017

Received in revised form

24 July 2017

Accepted 25 July 2017

Available online 26 July 2017

\section{Keywords:}

Natural estrogens

Conjugates

Wastewater treatment plants

Operational parameters

Removal pathways

\begin{abstract}
A B S T R A C T
Natural estrogens (NEs) discharged from wastewater treatment plants (WWTPs) have drawn great attention because of their potential risks to aquatic ecosystems. However, neglect of the conjugated natural estrogens (C-NEs) has caused large discrepancies among different studies on the removal of NEs in WWTPs. The present work investigated the transformation and fate of three NEs and six corresponding C-NEs along wastewater treatment processes. The removal efficiencies of the target estrogens (i.e., NEs and C-NEs) and their correlations with the operational parameters were determined over a twelve-month monitoring period at a typical WWTP adopting a combined bio-treatment process (i.e., anaerobic/anoxic/oxic process followed by a moving-bed biofilm reactor). The concentration variations of the target estrogens along the treatment processes were examined to differentiate the transformation and fate of NEs and C-NEs. Moreover, lab-scale experiments were conducted to clarify the removal pathways of C-NEs in the bio-treatment process. Results indicate that both NEs and C-NEs could pass through the treatment processes, thus being frequently detected in the effluent and excess sludge. The aqueous removal efficiencies of NEs and C-NEs were significantly correlated with the sludge retention time and temperature, respectively. C-NEs were more persistent than NEs, so considerably high conjugated ratios (13.5-100.0\%) were detected in the effluent. Sulfate conjugates presented a lower adsorption affinity to sludge and a slower hydrolysis rate than glucuronide conjugates, which makes the former more recalcitrant to biodegradation. This study highlights the challenge on the elimination of NEs, particularly their conjugates, by wastewater treatment processes.
\end{abstract}

(ㅇ) 2017 Elsevier Ltd. All rights reserved.

\section{Introduction}

Since the 1990s, the occurrence of endocrine disrupting chemicals (EDCs) in the environment has induced great challenges to the safety of aquatic ecosystems and received increasing attention (Schwarzenbach et al., 2006; Ternes et al., 1999). Among the EDCs, natural estrogens (NEs), including estrone (E1), 17 -estradiol (E2) and estriol (E3), were the most studied because of their strong estrogenic potencies (Liu et al., 2015). Human and animal excretions constituted the main sources of NEs in the environment, while the occurrence of NEs in municipal wastewater is primarily attributed to human excretions. The occurrence and behavior of

\footnotetext{
* Corresponding author.

E-mail address: qiangz@rcees.ac.cn (Z. Qiang).
}

NEs in wastewater treatment plants (WWTPs) has been extensively investigated around the world (Hamid and Eskicioglu, 2012; Liu et al., 2009; Nie et al., 2012), but the reported NE removal efficiencies in different WWTPs are largely variable. An important reason for the inconsistencies is that the operational parameters, including temperature, hydraulic retention time (HRT) and sludge retention time (SRT), varied greatly among the different studies. However, studies that discussed the influence of operational parameters on the NE removal also showed significant discrepancies (Clara et al., 2005; Hashimoto et al., 2007; Liu et al., 2015; Nakada et al., 2006). It is particularly difficult to isolate the influence of one operational parameter, as the treatment processes and other operational parameters differed across these studies. Therefore, focusing only on one WWTP and statistically analyzing its longterm monitoring data may clarify the effects of different 
operational parameters.

Another reason behind the variations in the NE removal efficiencies found in previous studies is the neglect of conjugated natural estrogens (C-NEs). NEs are predominantly excreted from human and animal bodies in their conjugated forms, so C-NEs can constitute a notable proportion in the influent of WWTPs (Johnson and Williams, 2004). C-NEs can be deconjugated into their corresponding NEs by arylsulfatase and $\beta$-glucuronidase derived from microorganisms which are abundant in WWTPs (Dray et al., 1972; Liu et al., 2015), thus leading to an inaccurate estimation on the removal efficiencies of NEs. However, limited research has been conducted on the transformation and fate of C-NEs in full-scale WWTPs, probably due to a lack of easily applicable methods for simultaneous detection of both NEs and C-NEs in complicated matrices (Zhu et al., 2015). The C-NE removal pathways in biotreatment processes have been proposed as follows: the conjugates are first deconjugated into their corresponding NEs and then biodegraded (Gomes et al., 2009; Liu et al., 2015). However, Kumar et al. (2012) found that C-NEs were not converted stoichiometrically to their corresponding NEs in raw sewage, so other metabolites may be formed. Additionally, batch studies on the removal of C-NEs usually focused on the concentration variations of the NEs and C-NEs (D'Ascenzo et al., 2003; Gomes et al., 2009; Kumar et al., 2012), while the contributions of enzymatic hydrolysis, biodegradation and sludge adsorption have not yet been separately identified. Thus, the removal pathways of C-NEs in wastewater treatment processes need to be further clarified.

This work aimed to elucidate the transformation and fate of three NEs and six C-NEs in WWTPs. A typical WWTP was selected for long-term (twelve-month) monitoring, which adopted an anaerobic/anoxic/oxic process followed by a moving-bed biofilm reactor (A/A/O-MBBR) as bio-treatment. The aqueous removal efficiencies of target estrogens were determined and further correlated with the operational parameters of the WWTP. The difference in the transformation and fate of NEs and C-NEs was clarified by monitoring their concentration variations along the treatment processes. In particular, the removal pathways of C-NEs in the biotreatment were explored by lab-scale tests under well controlled conditions.

\section{Materials and methods}

\subsection{Chemicals}

NE (E1, E2, and E3) standards were purchased from Dr. Ehrenstorfer GmbH (Augsburg, Germany; purity > 99.0\%). Sodium salts of six C-NEs, including estrone 3- $\beta$-D-glucuronide (E1-3G), $\beta$-estradiol 3- $\beta$-D-glucuronide (E2-3G), $\beta$-estradiol 17- $\beta$-D-glucuronide (E2$17 \mathrm{G})$, estrone 3-sulfate (E1-3S), $\beta$-estradiol 3-sulfate (E2-3S), and estriol 3-sulfate (E3-3S), were purchased from Sigma-Aldrich (Shanghai, China; purity > 95\%). Two internal standards, E2- $\mathrm{d}_{2}$ ( $\geq 98 \%$ ) and $\mathrm{E} 2-3 \mathrm{~S}-\mathrm{d}_{4}$ (50\% Tris), were supplied by CDN Isotopes (Quebec, Canada). The major physicochemical properties of the target estrogens and internal standards are listed in Table S1. The stock solutions of the target estrogens and internal standards were individually prepared by dissolving each compound in methanol at a concentration of $1000 \mathrm{mg} \mathrm{L}^{-1}$ and then stored at $-20^{\circ} \mathrm{C}$ in a refrigerator.

Methanol, methyl $t$-butyl ether, acetonitrile, dichloromethane, ammonia (25\% aqueous solution, by weight) and acetone, all of high-performance liquid chromatography grade, were obtained from Fisher Scientific (Geel, Belgium). Na ${ }_{2}$ EDTA-McIlvaine buffer was prepared by dissolving $21.00 \mathrm{~g}$ citric acid monohydrate, $17.75 \mathrm{~g}$ $\mathrm{Na}_{2} \mathrm{HPO}_{4}$, and $60.50 \mathrm{~g} \mathrm{Na} 2 \mathrm{EDTA} \cdot 2 \mathrm{H}_{2} \mathrm{O}$ in $1.625 \mathrm{~L}$ of ultrapure water, with $\mathrm{pH}$ adjusted to $4.00 \pm 0.05$. $\beta$-Glucuronidase from Helix pomatia (containing $100,000 \mathrm{U} \mathrm{mL}^{-1}$ glucuronidase activity and $5000 \mathrm{U} \mathrm{mL}^{-1}$ sulfatase activity), saccharic acid 1,4-lactone monohydrate (SA, an inhibitor for $\beta$-glucuronidase), STX 64 (an inhibitor for sulfatase), D-glucuronic acid sodium, glucuronide phenolphthalein and $\mathrm{NaN}_{3}$, were all purchased from Sigma-Aldrich (Shanghai, China). Ultrapure water was produced from a Milli-Q system (Advantage A10, Millipore, USA).

\subsection{Sample collection}

The selected WWTP is located in Wuxi City, Jiangsu Province, East China, which serves 820,000 inhabitants and operates with a flow rate of $100,000 \mathrm{~m}^{3} \mathrm{~d}^{-1}$. The treatment processes comprise a screen and a horizontal-flow grit chamber as the primary treatment, an A/A/O-MBBR combined bio-treatment process as the secondary treatment, and rotary fiber disc filters and UV disinfection as the tertiary treatment. A twelve-month monitoring investigation was conducted from July 2014 to June 2015. The influent, effluent and excess sludge samples were collected every month, and concurrently the real-time operational parameters, including HRT, SRT and temperature, were recorded (Table S2). During the sampling periods, major characteristic parameters of the influent and effluent (Table S3) were analyzed to confirm the normal operation of the WWTP. Additionally, flow-proportional (24 h) composite samples were collected using automatic samplers (SD900, Hach, Loveland, USA) from each treatment unit along the treatment processes in July 2015, with the sampling points illustrated in Fig. 1. All the acquired samples were subjected to pretreatment and extraction (detailed in Section 2.3) on site, and the resulting solid phase extraction (SPE) cartridges were stored in ice boxes and transported to our laboratory for analysis.

\subsection{Analytical methods and quality control}

The extraction and detection of the target estrogens in the wastewater and sludge samples followed the methods developed in our previous study (Zhu et al., 2015). In brief, SPE was applied to concentrate the target estrogens and purify the interfering matrix in the wastewater samples with an Oasis HLB cartridge coupled with a Sep-pak C18 cartridge ( $500 \mathrm{mg} / 6 \mathrm{~mL}$ both, Waters, USA). For the sludge samples, ultrasonic solvent extraction was first adopted to extract the target estrogens from the sludge before the SPE procedure. Subsequently, the target estrogens were separated using an Agilent 1290 ultra-performance liquid chromatography equipped with a Waters BEH-C18 column $(100 \mathrm{~mm} \times 2.1 \mathrm{~mm}, 1.7 \mu \mathrm{m})$, and detected by an Agilent 6420 Triple Quad mass spectrometer equipped with a negative electrospray ionization source in the multiple reaction monitoring mode. Quantification of the target estrogens was performed with the internal standard method for minimizing matrix effects. The recoveries were in the ranges of $61.2-122.4 \%$ and $59.7-106.2 \%$ for the wastewater and sludge, respectively; the limits of quantification (LOQs) ranged from 0.04 (E2-3S) to $1.4 \mathrm{ng} \mathrm{L}^{-1}$ (E2-17G) and from 0.05 (E2-3S) to $1.9 \mathrm{ng} \mathrm{g}^{-1}$ (E2) for the wastewater and sludge, respectively (Table S1). Calibration curves for the target estrogens were re-established every month. If any estrogen concentration was detected above the upper limit of the calibration range, then the sample was diluted and analyzed again. During sample analysis, ongoing quality control checks, including blank, duplicate of samples, and spiking recovery checks, were performed every 15 samples to ensure that all data were of good quality.

\subsection{Lab-scale batch experiments}

A series of lab-scale batch experiments were carried out to 


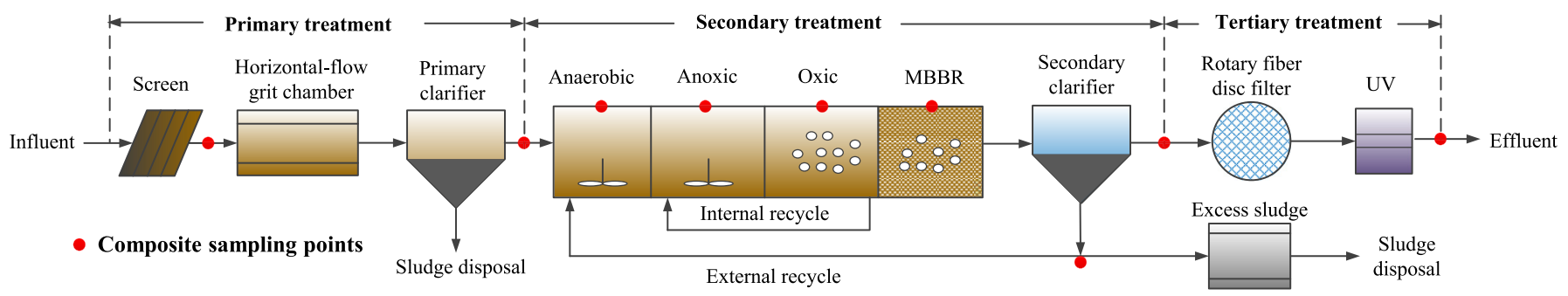

Fig. 1. Flow scheme of the wastewater treatment processes with sampling locations.

explore the removal pathways of C-NEs by taking E1-3G and E1-3S as model compounds. The mixed liquor ( $\mathrm{pH}=6.8$, MLSS (mixed liquor suspended solids) $=262 \mathrm{mg} \mathrm{L}^{-1}$ ) was taken from the oxic tank of the WWTP and used as the reaction matrix. To isolate the enzymatic hydrolysis of C-NEs from other removal pathways, SA and STX 64 were respectively adopted to inhibit the glucuronidase and sulfatase activity if necessary. In the preliminary experiments, the performance of SA and STX 64 in inhibiting the glucuronidase and sulfatase activity was confirmed. Moreover, the enzyme activity of the mixed liquor was tested, and the appropriate concentrations of the enzyme inhibitors spiked were determined accordingly. The detailed experimental procedures are presented in Text S1. The results indicate that the addition of $10 \mu \mathrm{M} \mathrm{U}^{-1}$ SA could completely inhibit the glucuronidase activity. Similarly, the addition of $10 \mu \mathrm{M} \mathrm{U}^{-1}$ STX 64 could completely inhibit the sulfatase activity. The total enzyme activity of the mixed liquor was detected as $0.33 \mathrm{U}$, so $10 \mu \mathrm{M}$ SA and STX 64 could sufficiently inhibit the enzymatic hydrolysis of E1-3G and E1-3S in the batch experiments, respectively.

Before spiking E1-3G and E1-3S, the mixed liquor (20 mL) was pre-treated as follows: 1) sterilized - heated at $121{ }^{\circ} \mathrm{C} ; 2$ ) filtered - passed through $0.22 \mu \mathrm{m}$ polyethersulfone (PES) filters; 3 ) inhibited - addition of $10 \mu \mathrm{M}$ SA or STX 64 to inhibit enzymatic hydrolysis; 4 ) azide - addition of $0.1 \% \mathrm{NaN}_{3}$ to inhibit microbial activity; and 5) original - without pre-treatment. Meanwhile, control experiments were carried out in ultrapure water with a target estrogen (E1-3G or E1-3S) spiked. All experiments were conducted in duplicate in $50 \mathrm{~mL}$ glass reactors with magnetically stirring at $150 \mathrm{rpm}$. The reactors were placed in a water bath with the temperature controlled at $37{ }^{\circ} \mathrm{C}$, which is optimal for the $\beta$ glucuronidase activity. The E1-3G or E1-3S stock solution (1000 $\mathrm{mg} \mathrm{L}^{-1}$ in methanol) was diluted by ultrapure water to prepare a working solution of $10 \mathrm{mg} \mathrm{L}^{-1}$. Then, a certain volume of the working solution (i.e., 939 and $743 \mu \mathrm{L}$ for E1-3G and E1-3S, respectively) was added to the pre-treated mixed liquor $(20 \mathrm{~mL})$ to reach an initial concentration of $1000 \mathrm{nM}$. Samples were withdrawn at pre-selected times, filtered through $0.22 \mu \mathrm{m}$ PES filters, and then analyzed.

\section{Results and discussion}

\subsection{Occurrence of the target estrogens in the influent, effluent and excess sludge}

During the twelve-month monitoring period, all the target estrogens were detected in the collected samples. Their detection frequencies and concentrations in the influent, effluent and excess sludge samples are shown in Fig. 2. In the influent samples, the NEs were all detected with frequencies of $100 \%$ and concentration ranges of 40.8-278.5, 4.6-42.1 and 15.1-364.9 $\mathrm{ng} \mathrm{L}^{-1}$ for E1, E2 and E3, respectively. Their maximum concentrations were all slightly higher than those reported in the WWTPs in Germany (Andersen

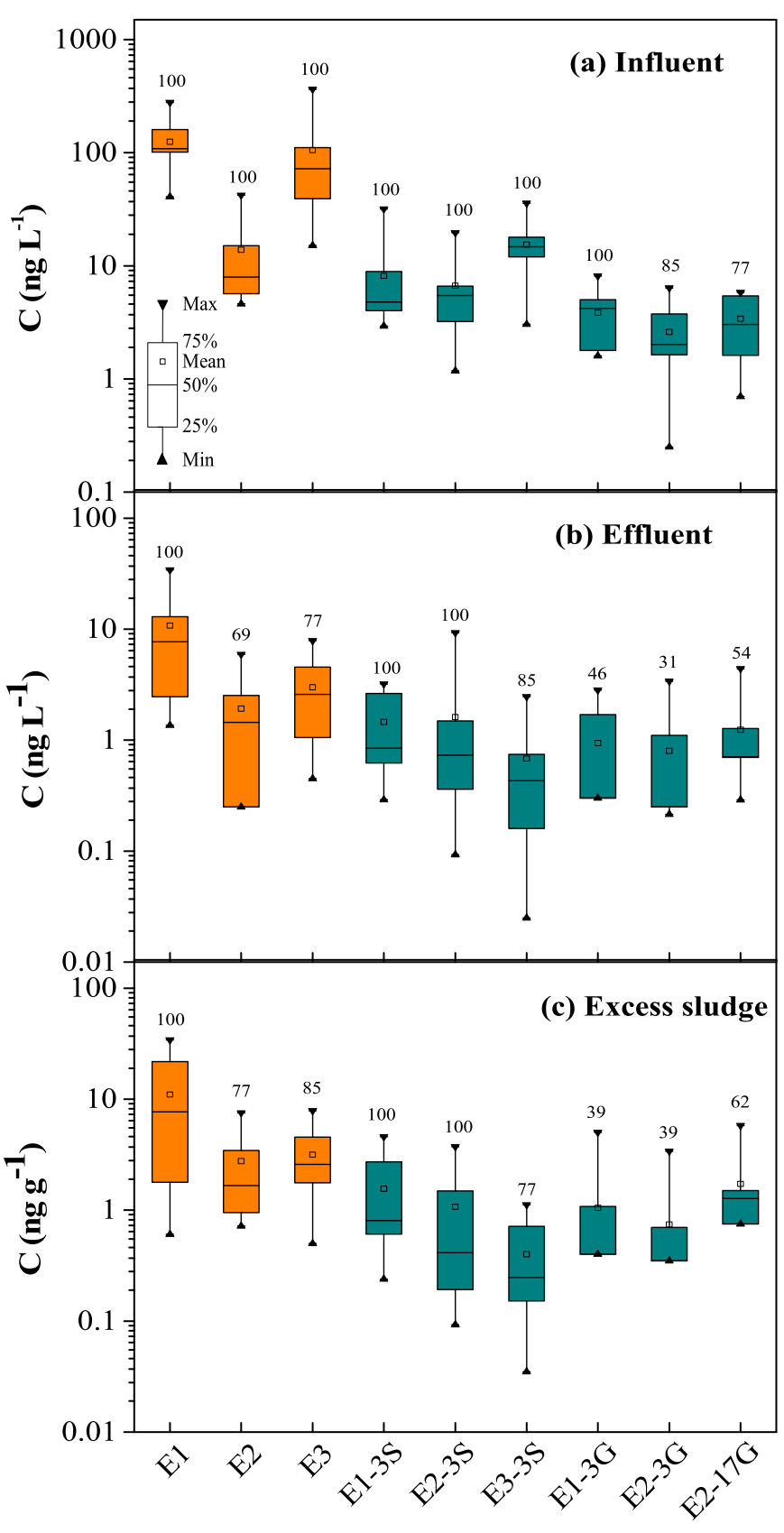

Fig. 2. Twelve-month monitoring concentrations of the target estrogens in the influent (a), effluent (b) and excess sludge (c) of the studied WWTP. The detection frequency (\%) of each estrogen is indicated numerically at the top. If a target estrogen was not detected in the collected samples $(<\mathrm{LOQ})$, half of the LOQ was applied as its concentration in the statistical analysis. The $50 \%$ line overlaps with the $25 \%$ line in the cases of E1-3G and E2-3G in the effluent and excess sludge. 
et al., 2003) and Japan (Kumar et al., 2015), but similar to the levels in other WWTPs in China (Huang et al., 2014; Xu et al., 2014). Regarding the C-NEs, the sulfate conjugates were all detected with frequencies of $100 \%$, with the median (i.e., the $50 \%$ line) concentrations of $4.8,5.5$ and $14.7 \mathrm{ng} \mathrm{L}^{-1}$ for E1-3S, E2-3S and E3-3S, respectively. Comparatively, lower detection frequencies were observed for the glucuronide conjugates, with the median concentrations of 4.2, 2.0 and $3.0 \mathrm{ng} \mathrm{L}^{-1}$ for E1-3G, E2-3G and E2-17G, respectively. In general, glucuronated estrogens were detected at lower frequencies and concentrations in WWTP influents than the NEs and sulfated estrogens (Liu et al., 2010), partly because the glucuronide forms are more readily deconjugated into free forms in sewage transport pipes where abundant $\beta$-glucuronidase is produced by fecal bacteria (Escherichia coli) (D'Ascenzo et al., 2003). Kumar et al. (2012) reported a half-life of $0.4 \mathrm{~h}$ for both E2-3G and E1-3G, $11.5 \mathrm{~h}$ for E2-3S, and $13.9 \mathrm{~h}$ for E1-3S in raw sewage in the sewer. Hence, complete absence of glucuronated estrogens in the influent can be expected in the case of a long sewage transport time.

In the effluent samples, the NEs remained as the dominant species with frequencies higher than $69 \%$ and median concentrations ranging from 1.4 (E2) to $7.7 \mathrm{ng} \mathrm{L}^{-1}$ (E1). The concentration levels were comparable to those in WWTP effluents reported in the literature (Andersen et al., 2003; Nie et al., 2009). The C-NEs were also commonly detected in the effluent samples, with frequencies of $85-100 \%$ and $31-54 \%$ for the sulfate and glucuronide conjugates, respectively. The median concentrations of the sulfate conjugates ranged from 0.4 (E3-3S) to $0.8 \mathrm{ng} \mathrm{L}^{-1}$ (E1-3S), while slightly lower median concentrations ranging from 0.3 (E1-3G) to 0.7 (E2-17G) $\mathrm{ng} \mathrm{L}^{-1}$ were observed for the glucuronide conjugates. Hence, all the target estrogens could potentially pass through the wastewater treatment processes, thus posing risks to receiving water bodies.

Adsorption by sludge is an important pathway for the elimination of micropollutants from wastewater. The target estrogens in the excess sludge samples were detected with frequencies similar to those in the effluent samples. The NEs were detected with the highest median concentrations (i.e., $1.7-7.7 \mathrm{ng} \mathrm{g}^{-1}$ ). The glucuronide conjugates showed slightly higher median concentrations (i.e., $0.4-1.3 \mathrm{ng} \mathrm{g}^{-1}$ ) than the sulfate conjugates (i.e., $0.3-0.8 \mathrm{ng} \mathrm{g}^{-1}$ ), which is different from that in the effluent. This result implies that the glucuronide conjugates were more likely to adsorb onto sludge particles than the sulfate conjugates. Limited field data are available related to glucuronated estrogens in the sludge phase in WWTPs. Hu et al. (2007) reported that E1-3S and E2-3S were adsorbed onto sludge in a WWTP, but no glucuronide conjugates were detected. The discrepancies among different studies suggest that further exploration of the adsorption behavior of sulfate and glucuronide conjugates by sludge is needed.

\subsection{Correlations of aqueous removal efficiencies of the target estrogens with operational parameters}

The aqueous removal efficiency $\left(R E_{\mathrm{aq}}\right)$, which defines the elimination of target estrogens from wastewater, is calculated as follows:

$R E_{\mathrm{aq}}(\%)=\frac{C_{\mathrm{inf}}-C_{\mathrm{eff}}}{C_{\mathrm{inf}}} \times 100$

where $C_{\text {inf }}$ and $C_{\text {eff }}\left(\mathrm{ng} \mathrm{L}^{-1}\right)$ are the concentrations of a target estrogen in the influent and effluent of the WWTP, respectively.

The $R E_{\mathrm{aq}}$ values of the target estrogens during the twelve-month monitoring period are presented in Fig. 3. E3 and E3-3S showed high and stable $R E_{\mathrm{aq}}$ values in the ranges of $68.4-100.0 \%$ (median

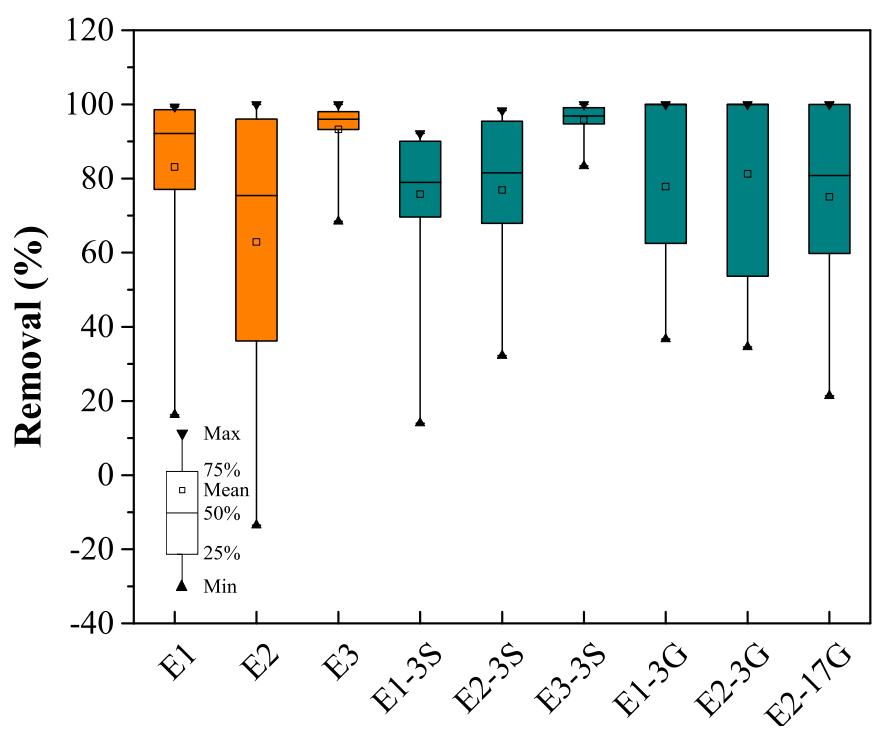

Fig. 3. Aqueous removal efficiencies of the target estrogens in a twelve-month monitoring period at the studied WWTP $(n=12)$. The $50 \%$ line overlaps with the $75 \%$ line in the cases of E1-3G and E2-3G.

96.0\%) and 83.4-100.0\% (median 96.9\%), respectively. E1 and E2 presented median $R E_{\text {aq }}$ values of $92.1 \%$ and $75.4 \%$, respectively. Similar removal efficiencies were observed for E1-3S and E2-3S, showing median $R E_{\mathrm{aq}}$ values of $78.9 \%$ and $81.5 \%$, respectively. The median $R E_{\mathrm{aq}}$ of both E1-3G and E2-3G was $100 \%$, indicating an efficient removal of glucuronide conjugates from wastewater by the treatment processes. However, wide inter-quartiles of the corresponding boxplots were observed for most of the target estrogens, which denotes considerable variations in their removal efficiencies over the twelve months. The operational parameters, including temperature, HRT and SRT, showed large fluctuations during the twelve-month monitoring period (Table S2), which may be embodied in the removal rates of the target estrogens. Therefore, statistical analysis using Pearson correlation was conducted to examine the relationship between the operational parameters and the $R E_{\mathrm{aq}}$ values of C-NEs and NEs. The results calculated by IBM SPSS Statistics software (V 19.0, USA) are summarized in Table 1. Note that E3 and E3-3S were excluded from the correlation analysis because of their high and stable $R E_{\mathrm{aq}}$ values.

SRT showed a significant correlation with the $R E_{\mathrm{aq}}$ values of NEs $\left(r^{2}=0.594, p<0.05\right)$, which agrees with the result of previous studies, that is, the removal of NEs was improved with increasing SRT in WWTPs (Kreuzinger et al., 2004; Kumar et al., 2015). A longer SRT allows the sludge to establish a more diverse biocoenosis and enrich slowly growing microorganisms (Clara et al., 2005) that may degrade the NEs (Kumar et al., 2015). Additionally, the relative abundance of hydrophobic genera also increases with the

Table 1

Pearson correlation analysis of the operational parameters with the aqueous removal efficiencies $\left(R E_{\mathrm{aq}}\right)$ of target estrogens.

\begin{tabular}{|c|c|c|c|c|}
\hline & \multicolumn{2}{|c|}{$R E_{\mathrm{aq}}$ of $\mathrm{NEs}{ }^{\mathrm{a}}$} & \multicolumn{2}{|c|}{$R E_{\mathrm{aq}}$ of $\mathrm{C}-\mathrm{NEs} \mathrm{s}^{\mathrm{b}}$} \\
\hline & $r^{2}$ & $p$ & $r^{2}$ & $p$ \\
\hline Temperature & 0.148 & 0.630 & 0.731 & 0.005 \\
\hline HRT & 0.034 & 0.912 & 0.443 & 0.130 \\
\hline SRT & 0.594 & 0.032 & 0.432 & 0.141 \\
\hline
\end{tabular}

a NEs: natural estrogens including E1 and E2.

b C-NEs: conjugated natural estrogens including E1-3G, E2-3G, E2-17G, E1-3S and E2-3S. 
sludge age, which tends to enhance the absorption capacity of the biomass for the NEs (Davenport et al., 2000).

The $R E_{\mathrm{aq}}$ values of C-NEs were significantly related to temperature $\left(r^{2}=0.731, p<0.01\right)$. Koh et al. (2009) found that a temperature reduction of $6{ }^{\circ} \mathrm{C}$ decreased the removal of E1-3S by $20 \%$ in two full-scale WWTPs. Similarly, a strong correlation was established between increasing temperature and decreasing conjugate concentration in the aqueous phase of activated sludge $\left(r^{2}=0.994\right)$ (Gomes et al., 2009). Temperature can influence the activity of arylsulfatase and $\beta$-glucuronidase as well as the abundance of bacteria that contain these enzymes (Liu et al., 2015; Nakada et al., 2006), which in turn affects the deconjugation of C-NEs. Table 1 shows that HRT and SRT had little correlation with the removal of C-NEs $(p>0.05)$, which indirectly supports that enzyme hydrolysis was a major removal pathway of C-NEs.

\subsection{Transformation and fate of the target estrogens in the WWTP}

The transformation of target estrogens along the wastewater treatment processes is shown in Fig. 4. The total concentrations of E1, E2 and E3 (i.e., sum of the free and conjugated forms of each estrogen) decreased with successive treatments through the WWTP. The primary treatment (i.e., the screen and horizontal-flow grit chamber) removed the total estrogens from wastewater by $9.8 \%$ (total E2) to $26.8 \%$ (total E3). Then, the A/A/O-MBBR combined biotreatment process accounted for the major removal of the target estrogens. However, when divided into individual treatment units, the anaerobic unit had a low potency for removing the target

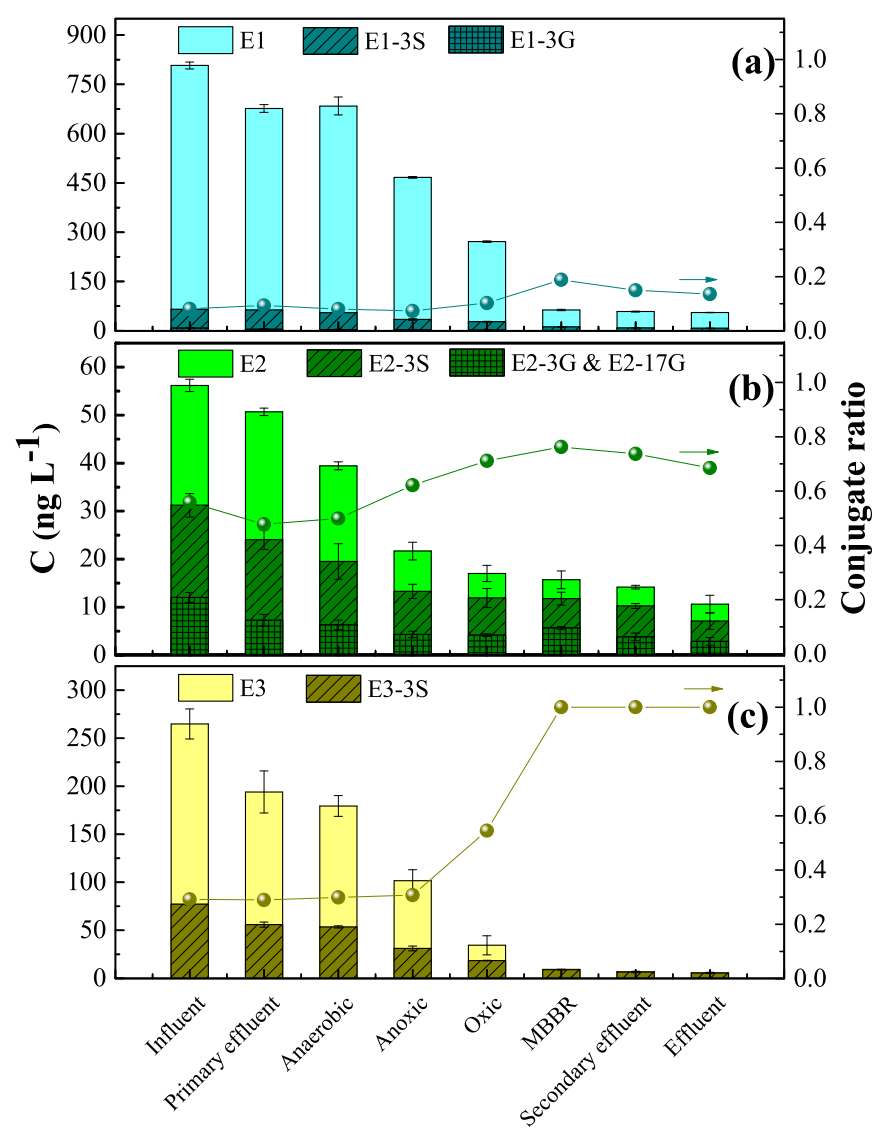

Fig. 4. Variations in the concentrations and conjugate ratios of E1 (a), E2 (b) and E3 (c) along the wastewater treatment processes. For each estrogen, the conjugate ratio is calculated as the conjugate concentration divided by the total estrogen concentration (i.e., sum of the free and conjugated forms). estrogens from wastewater. The total E1 concentration even showed a slight increase in the anaerobic unit. Because E2 is biodegraded via E1 (Shi et al., 2004), the increasing E1 concentration was probably due to the fact that the generation rate of E1 exceeded its removal rate. In contrast, the total estrogens significantly decreased along the anoxic, oxic and MBBR units. Similarly, Andersen et al. (2003) reported that the denitrification and aerated tanks in a WWTP were the most important treatment units for removing estrogens. Joss et al. (2004) also found that the maximum removal rate of E1 occurred under aerobic conditions in WWTPs. The biodegradation of estrogens by activated sludge was considered as dominated by the co-metabolism of ammonia oxidizing bacteria (Ren et al., 2007). In this case, the oxidation treatment units would certainly show a better performance in removing estrogens.

For each estrogen (i.e., E1, E2 or E3), the conjugate ratio is calculated as the conjugate concentration divided by the total estrogen concentration (i.e., sum of the free and conjugated forms). The variations in the conjugate ratios along the treatment processes can reflect the difference in the transformation and fate of NEs and C-NEs. Fig. 4 shows that the conjugates occupied $8.1 \%, 55.9 \%$ and $29.2 \%$ of the total E1, E2 and E3 in the influent, respectively. The conjugate ratio gradually increased along the bio-treatment units and reached the peak values of $18.8 \%, 76.3 \%$ and $100 \%$ for E1, E2 and E3 in the MBBR, respectively. Because the deconjugation of C-NEs into NEs was expected to be continuous in the bio-treatment, the increasing conjugate ratio implies that the elimination of NEs could be faster than the transformation of C-NEs into NEs. After the combined bio-treatment process, the conjugate ratios of E1 and E2 slightly decreased, probably because the enzymatic hydrolysis of CNEs continued whereas the biodegradation of NEs almost stopped in the water phase. The conjugate ratios of E1, E2, and E3 in the final effluent reached $13.5 \%, 68.5 \%$ and $100.0 \%$, respectively, which were much higher than those in the influent. Because certain conjugated estrogens (e.g., E3-3G and E2-17S) were not analyzed due to the lack of standards, the conjugate ratios may be even higher than the calculated values. Therefore, the more persistence of C-NEs emphasizes that they could follow different removal pathways from NEs, which needs to be further clarified.

\subsection{Removal pathways of the conjugated estrogens}

As stated above, the deconjugation of C-NEs through enzymatic hydrolysis is regarded as an important removal pathway of C-NEs in the bio-treatment process (Gomes et al., 2009; Liu et al., 2015). In addition, the strong correlation of the C-NE removal with temperature also highlights the importance of enzymatic hydrolysis. However, biodegradation and sludge adsorption may also remove the C-NEs from wastewater, but little information is available about their contributions. In this work, efficient inhibitors for $\beta$-glucuronidase and sulfatase were applied to preclude the enzymatic hydrolysis pathway.

As shown in Fig. 5a, in the sterilized group, E1-3G was removed by $25 \%$ while no E1 was generated within $4 \mathrm{~h}$. Because both the enzymatic and microbial activities were inhibited, the loss of E13G from the water phase could only be attributed to sludge adsorption, which reached equilibrium after approximately $40 \mathrm{~min}$. In the filtered group, the E1-3G concentration kept decreasing until a $90 \%$ reduction was reached at $4 \mathrm{~h}$. Meanwhile, E1 was continuously formed, and the summed concentration of E1-3G and E1 over the reaction course was almost equal to the initial concentration of E1-3G (i.e., $1000 \mathrm{nM}$ ). Therefore, the transformation of E1-3G into E1 by enzymatic hydrolysis represented a unique removal pathway in the absence of sludge. In addition, this result indicates that hydrolytic enzymes were 

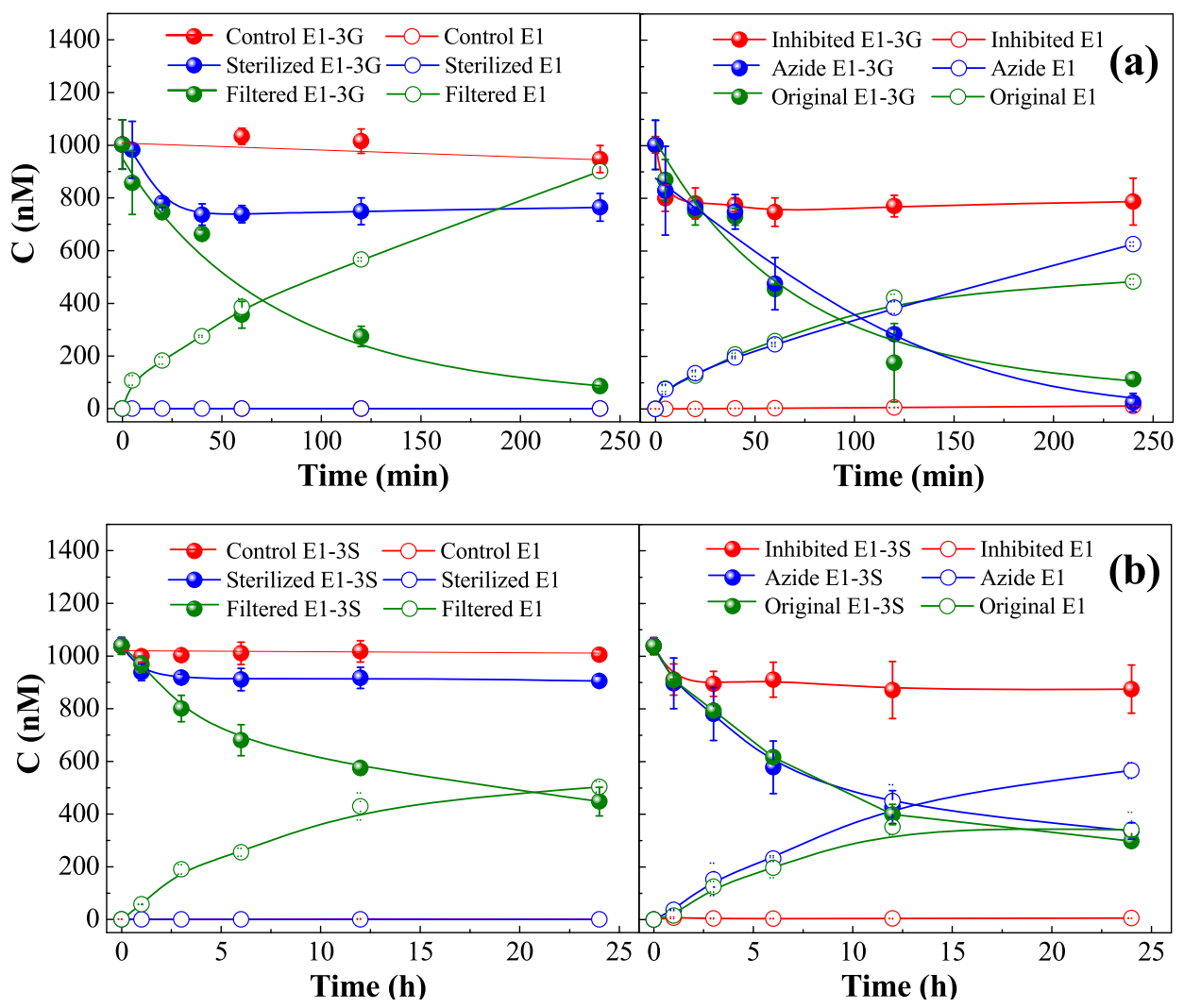

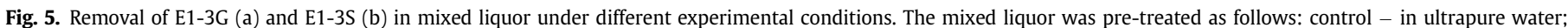

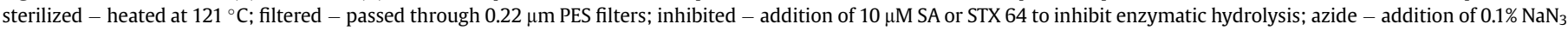
to inhibit microbial activity; original - without pre-treatment. Error bars represent the relative percent difference of duplicate experiments.

present in the filtrate and enzymatic hydrolysis was independent of the presence of biomass.

With regard to the inhibited group, the removal of E1-3G was similar to that in the sterilized group, where sludge adsorption was the only removal pathway. Even though the enzymatic activity was inhibited, the biomass was still active in the inhibited group. However, no improvement in the E1-3G removal indicates that the direct biodegradation of E1-3G by biomass was impossible. In the original and azide groups, the removal of E1-3G showed similar trends, with E1 generated concurrently. The summed concentration of E1-3G and E1 were 597 and $650 \mathrm{nM}$ at $4 \mathrm{~h}$ in the original and azide groups, respectively. Because the microbial activity was completely inhibited in the azide group, the relatively lower concentration in the original group suggests that subsequent biodegradation occurred for the newly-generated $\mathrm{E} 1$.

The trends for E1-3S removal and E1 generation in different experimental groups (Fig. 5b) were similar to those of E1-3G, indicating that E1-3S had the same removal pathways as E1-3G in the bio-treatment. However, E1-3S exhibited a considerably lower adsorption rate and capacity than E1-3G. In the sterilized group, sludge adsorption reached equilibrium at $5 \mathrm{~h}$, which only caused a $10 \%$ reduction in the E1-3S concentration. In the filtered group, the E1-3S concentration decreased by $56 \%$ after $24 \mathrm{~h}$, which was significantly slower than that of E1-3G (i.e., 90\% after $4 \mathrm{~h}$ ), indicating a weaker enzymatic activity for E1-3S hydrolysis. In a laboratory test, D'Ascenzo et al. (2003) found that the complete deconjugation of E3-16G and E2-17G was achieved within one day, while the deconjugation of E1-3S and E2-3S was completed after 6 days due to a scarce arylsulfatase activity in domestic wastewater. Moreover, the more persistence of sulfate conjugates than glucuronide ones in sewer and river water was also reported (Kumar et al., 2012). The slower enzymatic hydrolysis rate was primarily responsible for the more persistence of sulfate conjugates in different matrices.

Based on the experimental results obtained above, the removal pathways of C-NEs in the bio-treatment are proposed as follows: 1) a small proportion of C-NEs were directly adsorbed by sludge; and 2) a large proportion of C-NEs were deconjugated into their corresponding NEs through enzymatic hydrolysis, which were then removed via sludge adsorption and biodegradation. The glucuronide conjugates presented a higher adsorption affinity to sludge and a faster hydrolysis rate than the sulfate conjugates, which accounted for their relatively higher aqueous removal efficiencies (Fig. 3). For each C-NE, its mass proportions in the effluent, in the excess sludge, and biodegraded as related to the total input mass in the influent were calculated according to Text S2. Fig. S1 shows that the biodegraded mass proportions were $97-99 \%$ for the glucuronide conjugates and $47-88 \%$ for the sulfate conjugates, which is primarily explained by the faster hydrolysis rate of the glucuronide conjugates. The mass proportions in the excess sludge were only $1-3 \%$ for the glucuronide conjugates and $5-31 \%$ for the sulfate conjugates. Although the sulfate conjugates had a lower adsorption affinity to sludge than the glucuronide conjugates, sludge adsorption still played an important role in their removal from wastewater in WWTPs.

\section{Conclusions}

This work investigated the transformation and fate of NEs and their conjugates (i.e., C-NEs) along the wastewater treatment 
processes. Based on both field investigation and lab-scale tests, the following conclusions are drawn:

- Substantial amounts of NEs and C-NEs could pass through wastewater treatment processes, thus being discharged through the effluent and excess sludge.

- The aqueous removal efficiencies of NEs and C-NEs were significantly correlated with the SRT and temperature, respectively; which corresponded to the removal pathways of biodegradation and enzymatic hydrolysis, respectively.

- C-NEs were removed from wastewater through enzymatic hydrolysis and sludge adsorption in the bio-treatment. Sulfate conjugates were more persistent than glucuronide conjugates in the WWTP, thus deserving more attention in the future.

\section{Acknowledgements}

This work was supported by the National Natural Science Foundation of China $(51525806,51420105012,21277160)$ and Chinese Academy of Sciences (ZDRW-ZS-2016-5-6).

\section{Appendix A. Supplementary data}

Supplementary data related to this article can be found at http:// dx.doi.org/10.1016/j.watres.2017.07.065.

\section{References}

Andersen, H.R., Siegrist, H., Halling-Sorensen, B., Ternes, T.A., 2003. Fate of estrogens in a municipal sewage treatment plant. Environ. Sci. Technol. 37 (18), $4021-4026$

Clara, M., Kreuzinger, N., Strenn, B., Gans, O., Kroiss, H., 2005. The solids retention time-a suitable design parameter to evaluate the capacity of wastewater treatment plants to remove micropollutants. Water Res. 39 (1), 97-106.

D'Ascenzo, G., Corcia, A.D., Gentili, A., Mancini, R., Mastropasqua, R., Nazzari, M. Samperi, R., 2003. Fate of natural estrogen conjugates in municipal sewage transport and treatment facilities. Sci. Total Environ. 302 (1-3), 199-209.

Davenport, R.J., Curtis, T.P., Goodfellow, M., Stainsby, F.M., Bingley, M., 2000 Quantitative use of fluorescent in situ hybridization to examine relationships between mycolic acid-containing actinomycetes and foaming in activated sludge plants. Appl. Environ. Microbiol. 66, 1158-1166.

Dray, J., Dray, F., Tiller, F., Ulman, A., 1972. Hydrolysis of urine metabolites of different steroid hormones by (-glucuronidase) from Escherichia coli. Ann. Ist. Pasteur. 123, 853-857.

Gomes, R.L., Scrimshaw, M.D., Lester, J.N., 2009. Fate of conjugated natural and synthetic steroid estrogens in crude sewage and activated sludge batch studies. Environ. Sci. Technol. 43 (10), 3612-3618.

Hamid, H., Eskicioglu, C., 2012. Fate of estrogenic hormones in wastewater and sludge treatment: a review of properties and analytical detection techniques in sludge matrix. Water Res. 46 (18), 5813-5833.

Hashimoto, T., Onda, K., Nakamura, Y., Tada, K., Miya, A., Murakami, T., 2007. Comparison of natural estrogen removal efficiency in the conventional activated sludge process and the oxidation ditch process. Water Res. 41, 2117-2126.

Hu, J.Y., Chen, X., Tao, G., Kekred, K., 2007. Fate of endocrine disrupting compounds in membrane bioreactor systems. Environ. Sci. Technol. 41, 4097-4102.

Huang, B., Li, X., Sun, W., Ren, D., Liu, Y., Li, Q., Pan, X., 2014. Occurrence, removal, and fate of progestogens, androgens, estrogens, and phenols in six sewage treatment plants around Dianchi Lake in China. Environ. Sci. Pollut. Res. 21 (22) $12898-12908$.

Johnson, A.C., Williams, R.J., 2004. A model to estimate influent and effluent concentrations of estradiol, estrone, and ethinylestradiol at sewage treatmen works. Environ. Sci. Technol. 38, 3649-3658.

Joss, A., Andersen, H.R., Ternes, T., Richle, P.R., Siegrist, H., 2004. Removal of estrogens in municipal wastewater treatment under aerobic and anaerobic conditions: consequences for plant optimization. Environ. Sci. Technol. 38, 3047-3055.

Koh, Y.K.K., Chiu, T.Y., Boobis, A.R., Scrimshaw, M.D., Bagnall, J.P., Soares, A., Pollard, S., Cartmell, E., Lester, J.N., 2009. Influence of operating parameters on the biodegradation of steroid estrogens and nonylphenolic compounds during biological wastewater treatment processes. Environ. Sci. Technol. 43 (17), 6646-6654.

Kreuzinger, N., Clara, M. Strenn, B., Kroiss, H., 2004. Relevance of the sludge retention time (SRT) as design criteria for wastewater treatment plants for the removal of endocrine disruptors and pharmaceuticals from wastewater. Water Sci. Technol. 50 (5), 149-156.

Kumar, V., Johnson, A.C., Nakada, N., Yamashita, N., Tanaka, H., 2012. De-conjugation behavior of conjugated estrogens in the raw sewage, activated sludge and river water. J. Hazard. Mater. 227-228, 49-54.

Kumar, V., Nakada, N., Yamashita, N., Johnson, A.C., Tanaka, H., 2015. Influence of hydraulic retention time, sludge retention time, and ozonation on the removal of free and conjugated estrogens in Japanese activated sludge treatment plants. CLEAN Soil Air Water 43 (9), 1289-1294.

Liu, Z.H., Hashimoto, T., Okumura, Y., Kanjo, Y., Mizutani, S., 2010. Simultaneous analysis of natural free estrogens and their conjugates in wastewater by GC-MS. CLEAN Soil Air Water 38 (2), 181-188.

Liu, Z.H., Kanjo, Y. Mizutani, S., 2009. Removal mechanisms for endocrine disrupting compounds (EDCs) in wastewater treatment - physical means, biodegradation, and chemical advanced oxidation: a review. Sci. Total Environ. 407 (2), 731-748.

Liu, Z.H., Lu, G.N., Yin, H., Dang, Z., Rittmann, B., 2015. Removal of natural estrogens and their conjugates in municipal wastewater treatment plants: a critical review. Environ. Sci. Technol. 49 (9), 5288-5300.

Nakada, N., Yasojima, M., Okayasu, Y., Komori, K., Tanaka, H., Suzuki, Y., 2006. Fate of oestrogenic compounds and identification of oestrogenicity in a wastewater treatment process. Water Sci. Technol. 53, 51-63.

Nie, Y.F., Qiang, Z.M., Zhang, H.Q., Adams, C., 2009. Determination of endocrinedisrupting chemicals in the liquid and solid phases of activated sludge by solid phase extraction and gas chromatography-mass spectrometry. J. Chromatogr. A 1216 (42), 7071-7080.

Nie, Y.F., Oiang, Z.M., Zhang, H.Q., Ben, W.W., 2012. Fate and seasonal variation of endocrine-disrupting chemicals in a sewage treatment plant with $\mathrm{A} / \mathrm{A} / \mathrm{O}$ process. Sep. Purif. Technol. 84, 9-15.

Ren, Y.X., Nakano, K., Nomura, M., Chiba, N., Nishimura, O., 2007. Effects of bacterial activity on estrogen removal in nitrifying activated sludge. Water Res. 41 (14), 3089-3096.

Schwarzenbach, R.P., Escher, B.I., Fenner, K., Hofstetter, T.B., Johnson, C.A., von Gunten, U., Wehrli, B., 2006. The challenge of micropollutants in aquatic systems. Science 313 (5790), 1072-1077.

Shi, J., Fujisawa, S., Nakai, S., Hosomi, M., 2004. Biodegradation of natural and synthetic estrogens by nitrifying activated sludge and ammonia-oxidizing bacterium Nitrosomonas europaea. Water Res. 38, 2323-2330.

Ternes, T.A., Stumpf, M., Mueller, J., Haberer, K., Wilken, R.D., Servos, M., 1999. Behavior and occurrence of estrogens in municipal sewage treatment plants - I. Investigations in Germany, Canada and Brazil. Sci. Total Environ. 225 (1), 81-90.

Xu, Y., Xu, N., Llewellyn, N.R., Tao, H., 2014. Occurrence and removal of free and conjugated estrogens in wastewater and sludge in five sewage treatment plants. Environ. Sci. Process. Impacts 16 (2), 262-270.

Zhu, B., Ben, W.W., Yuan, X.J., Zhang, Y., Yang, M., Qiang, Z.M., 2015. Simultaneous detection of endocrine disrupting chemicals including conjugates in municipal wastewater and sludge with enhanced sample pretreatment and UPLC-MS/MS Environ. Sci. Process. Impacts 17 (8), 1377-1385. 